\title{
UNCERTAINTY WITHIN ECONOMIC MODELS
}




\section{World Scientific Series in Economic Theory}

(ISSN: 2251-2071)

Series Editor: $\quad$ Eric Maskin (Harvard University, USA)

\section{Published}

Vol. 1 Equality of Opportunity: The Economics of Responsibility by Marc Fleurbaey and François Maniquet

Vol. 2 Robust Mechanism Design: The Role of Private Information and Higher Order Beliefs Dirk Bergemann and Stephen Morris

Vol. 3 Case-Based Predictions: An Axiomatic Approach to Prediction, Classification and Statistical Learning Itzhak Gilboa and David Schmeidler

Vol. 4 Simple Adaptive Strategies: From Regret-Matching to Uncoupled Dynamics Sergiu Hart and Andreu Mas-Colell

Vol. 5 The Language of Game Theory: Putting Epistemics into the Mathematics of Games Adam Brandenburger

Vol. 6 Uncertainty within Economic Models Lars Peter Hansen and Thomas J Sargent

\section{Forthcoming}

\section{Decision Theory}

Wolfgang Pesendorfer (Princeton University, USA) \&

Faruk Gul (Princeton University, USA)

\section{Leverage and Default}

John Geanakoplos (Yale University, USA)

\section{Leverage Cycle, Equilibrium and Default}

Vol. 2: Collateral Equilibrium and Default

John Geanakoplos (Yale University, USA)

\section{Learning and Dynamic Games}

Dirk Bergemann (Yale University, USA) \& Juuso Valimaki (Aalto University, Finland) 
World Scientific Series in Economic Theory - Vol. 6

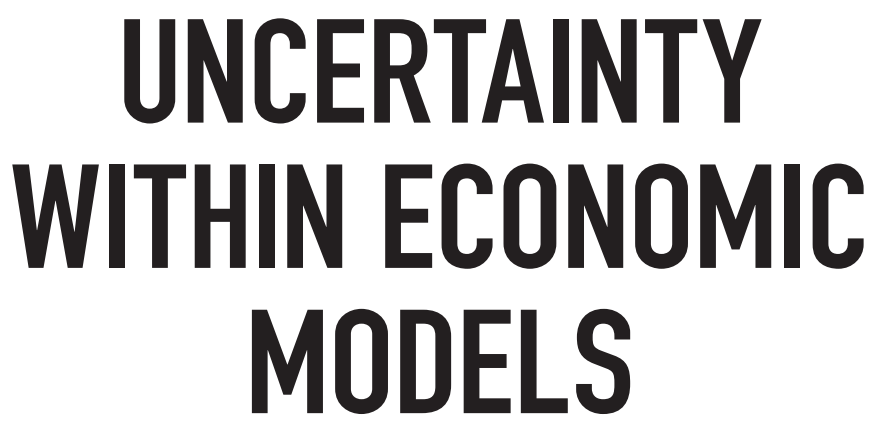

\section{Lars Peter Hansen}

University of Chicago, USA

\section{Thomas J Sargent}

New York University, USA \& Hoover Institution, USA 


\section{Published by}

World Scientific Publishing Co. Pte. Ltd.

5 Toh Tuck Link, Singapore 596224

USA office: 27 Warren Street, Suite 401-402, Hackensack, NJ 07601

UK office: 57 Shelton Street, Covent Garden, London WC2H 9HE

\section{Library of Congress Cataloging-in-Publication Data \\ Hansen, Lars Peter.}

Uncertainty within economic models / by Lars Peter Hansen (University of Chicago, USA \&

The National Bureau of Economic Research, USA) and Thomas J Sargent (New York University,

USA \& Hoover Institution, USA). -- 1 Edition.

pages $\mathrm{cm}$.-- (World scientific series in economic theory ; 6)

Includes bibliographical references and index.

ISBN 978-9814578110 (hardcover) -- ISBN 9814578118 (hardcover)

1. Economics--Mathematical models. I. Sargent, Thomas J. II. Title.

HB135.H368 2014

$330.01 ' 5195--d c 23$

2014017341

\section{British Library Cataloguing-in-Publication Data}

A catalogue record for this book is available from the British Library.

Copyright $(C) 2015$ by World Scientific Publishing Co. Pte. Ltd.

All rights reserved. This book, or parts thereof, may not be reproduced in any form or by any means, electronic or mechanical, including photocopying, recording or any information storage and retrieval system now known or to be invented, without written permission from the publisher.

For photocopying of material in this volume, please pay a copying fee through the Copyright Clearance Center, Inc., 222 Rosewood Drive, Danvers, MA 01923, USA. In this case permission to photocopy is not required from the publisher.

In-house Editor: Alisha Nguyen

Typeset by Stallion Press

Email: enquiries@stallionpress.com

Printed in Singapore 
To David Jacobson and Peter Whittle 
This page intentionally left blank 


\section{Foreword}

Assuming "rational expectations" — that agents within a model and the econometrician observing those agents have specified the model correctly has proved to be an extraordinarily useful approach to macroeconomics, and Lars Hansen and Thomas Sargent have been in the vanguard of that approach. But Hansen and Sargent have also led the way in showing how departures from rational expectations - specifically, the possibility of model misspecification - can fruitfully be studied by macroeconomists.

In this volume, Hansen and Sargent have assembled ten fundamental articles on how to proceed when agents and econometricians are uncertain about the correct model. Nine of the ten are joint work (sometimes with additional authors); one is by Hansen alone. Hansen and Sargent have also written an introduction that lays out how they deal with misspecification in general and what the individual papers do in particular.

I am extremely grateful to Lars Hansen and Thomas Sargent for producing this important book. The profession will be grateful to them too.

Eric Maskin

Editor-in-Chief

World Scientific Series in Economic Theory 
This page intentionally left blank 


\section{Preface}

We have devoted large parts of our professional lives to refining and applying rational expectations macroeconomics and econometrics. This book describes some of our recent efforts to come to grips with misspecification about and within economic models, a problem that the rational expectations assumption ignores.

Rational expectations is a powerful device for restricting data. It eliminates all free parameters describing beliefs about endogenous outcomes and imposes a pervasive "communism of beliefs" among all agents within a model, the outside observers and econometricians estimating the model, and the "god" or "nature" that generates the actual data. Applied researchers exploit that communism of beliefs both in computing a rational expectations equilibrium and in constructing good statistical estimators based on likelihood functions and other moment matching methods.

Thirty five years of applying rational expectations econometrics delivered its share of disappointments and "creative destructions." The cross-equation restrictions that are a hall mark of rational expectations models have often generated convincing empirical evidence against a variety of ex ante beautiful theoretical models. One constructive response has been to sort through the wreckage left by those likelihood ratios and related statistical tests in search of diagnostics that hint at improved specifications of the preferences, technologies, timing protocols, and information flows that comprise the elements of a rational expectations model.

There have been other sensible responses. Our good friend Robert E. Lucas, Jr. told us in the early 1980s that our likelihood ratio tests and moment matching tests were rejecting too many good models. He went on to say that all models are at best approximations (a polite way of saying that they are wrong) that should be compared to the data with more forgiving methods like the calibration techniques advocated by Kydland and Prescott. Many macroeconomists have followed that advice. 
Specification searches accept uncomfortable departures from part of the "communism of beliefs" inherent in rational expectations econometrics. Modern specification searches typically hop from one rational expectations model to another, each model having its own "communism of beliefs" that precludes the agents within a model from entertaining specification doubts. The rational expectations assumption will not accommodate a completely coherent exploration of model misspecification.

As applied macroeconomists and time series econometricians, we have had to live with repeated messages that our models have failed specification tests. We are both fortunate to have been at the University of Minnesota during the 1970s, a time when Christopher A. Sims was formulating constructive responses to model misspecification. In a string of papers cast in terms of pre-rational expectations models (often distributed lags), Sims $(1971 \mathrm{a}, \mathrm{b}, 1972,1974)$ characterized adverse consequences of various misspecifications and provided recommendations about how to filter data or redesign approximating models in ways to protect against some types of misspecifications. ${ }^{1}$ Sims's papers prominently featured relative entropy, an expected log likelihood ratio of a misspecified joint probability to a "true" joint probability evaluated with respect the true probability. Importing insights of Kullback and Leibler (1951) into economics, Sims described how in large samples a class of good econometric estimators would minimize relative entropy or an equivalent quantity that Sims represented in the frequency domain. Sims used that approximation criterion to provide advice about how to adjust admittedly approximate specifications or to filter data in order to improve estimates of model features of special interest. We discern a robustness perspective underneath Sims's practical advice, since Sims's advice provides procedures that work "well enough" despite a researcher's admitting that his or her model is only an approximation.

Until the early 1990s, we were busy adapting and applying mathematical tools for dynamic optimization and prediction that had been perfected by mathematicians in the 1950s and 1960s. Those tools assume that a decision maker completely trusts a stochastic specification, which makes them naturally applicable to rational expectations models. While we struggled with specification doubts about our models, we maintained the assumption that the agents within our rational expectations models did not.

\footnotetext{
${ }^{1}$ Sims (1993) and Hansen and Sargent (1993) used related analytical methods to study consequences of misspecification in the context of rational expectations models.
} 
This was the price of admission for using those beautiful mathematical tools.

About 1992, by reading Jacobson (1973) and Whittle (1990) we learned that while we had been busy applying 1950s-1960s control and prediction theories for fully trusted models, mathematicians and engineers in the 1970s and 1980s had worked out "post-modern" control and prediction theories applicable to situations in which decision makers distrust their models. This work goes under a variety of names like robust control, $H$-infinity control, or minimum-entropy control. Having been prepared by our own repeated specification struggles, we immediately recognized that robust control and prediction tools could help us build macroeconomic models having agents within them who forecast and make decisions despite having specification doubts. That is how we began. 
This page intentionally left blank 


\section{Acknowledgements}

We thank our coauthors, Thomas Tallarini, Evan Anderson, Gauhar Turmuhambetova, Noah Williams, and Francisco Barillas, for working with us originally and for allowing us to reproduce our joint work here. We especially thank François Velde for extraordinary help in designing and typesetting this book. We thank Alisha Nguyen for guiding us through the process of preparing the manuscript and Eric Maskin and Zvi Ruder for suggesting that we prepare this book.

We thank their original publishers for allowing us to reprint versions of the following papers that form successive chapters of this book:

- Chapter 2, "Discounted Linear Exponential Quadratic Gaussian Control," IEEE Transactions on Automatic Control, May 1995, 40(5):968971;

- Chapter 3, "Robust Permanent Income and Pricing" (with Thomas Tallarini, Jr.), Review of Economic Studies, October 1999, 66(4): 873-907;

- Chapter 4, "A Quartet of Semigroups for Model Specification, Robustness, Prices of Risk, and Model Detection" (with Evan Anderson), Journal of the European Economic Association, March 2003, 1(1):68123 ;

- Chapter 5, "Robust Control and Model Uncertainty," American Economic Review, May 2001, 91(2):60-66;

- Chapter 6, "Robust Control and Model Misspecification" (with Gauhar Turmuhambetova and Noah Williams), Journal of Economic Theory, May 2006, 128(1):45-90;

- Chapter 7, "Doubts or Variability?" (with Francisco Barillas), Journal of Economic Theory, November 2009, 144(6):2388-2418;

- Chapter 8, "Recursive Robust Estimation and Control without Commitment," Journal of Economic Theory, September 2007, 136(1):1-27; 
- Chapter 9, "Fragile Beliefs and the Price of Uncertainty," Quantitative Economics, July 2010, 1(1):129-162;

- Chapter 10, "Beliefs, Doubts and Learning: Valuing Macroeconomic Risk" (by Lars Peter Hansen alone), American Economic Review, May 2007, 97(2):1-30;

- Chapter 11, "Three Types of Ambiguity," Journal of Monetary Economics, July 2012, 59(5):422-445. 


\section{About the Authors}

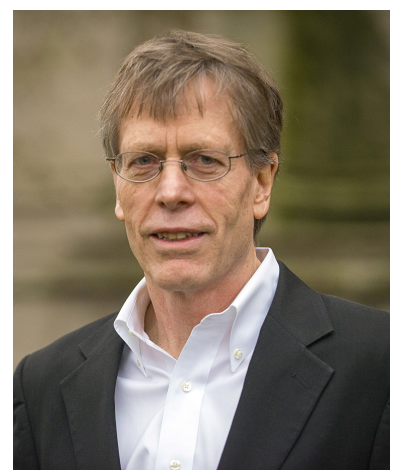

Lars Peter Hansen, the David Rockefeller Distinguished Service Professor at the University of Chicago, is an internationally known leader in economic dynamics. He was awarded the 2013 Nobel Prize in Economics, shared with Eugene F Fama (University of Chicago) and Robert J Shiller (Yale University), "for their empirical analysis of asset prices." Hansen guides the scholarly direction of the Becker Friedman Institute and chairs the Institute Research Council. He was one of the forces behind the 2008 creation of the Milton Friedman Institute, the predecessor of the Becker Friedman Institute, and served as its founding director.

Hansen's work explores formal implications of dynamic economic models in which decision makers face uncertain environments. The main theme of his research has been to devise and apply econometric methods that are consistent with the probabilistic framework of the economic models under investigation. His work has implications for consumption, savings investment, and asset pricing.

Hansen's early research in econometrics was aimed at developing time series statistical methods to investigate one part of an economic model without having to fully specify and estimate all of the model ingredients. The applications he explored with several coauthors included systems that are rich enough to support models of asset valuation and to identify and clarify empirical puzzles, where real-world financial and economic data were at odds with prevailing academic models. 
He continues to explore, analyze, and interpret implications of dynamic economic models in environments with uncertainty from a time-series perspective. His recent research explores ways to quantify intertemporal risk-return tradeoffs and ways to model economic behavior when decision makers are uncertain about how to forecast future economic events.

Hansen won the 2010 BBVA Foundation Frontiers of Knowledge Award in the Economics, Finance and Management "for making fundamental contributions to our understanding of how economic actors cope with risky and changing environments." He also received the CME GroupMSRI Prize in Innovative Quantitative Applications in 2008 and the Erwin PleinNemmers Prize in Economics from Northwestern University in 2006 .

He was selected to deliver the Princeton Lectures in Finance at the Bendheim Center for Finance in December 2010, the Tjalling C Koopmans Memorial Lectures at Yale in September 2008, and the Third Toulouse Lectures in Economics at the Université de Toulouse in May 2005.

Hansen is a fellow of the National Academy of Sciences and the American Finance Association. He also is a member of the American Academy of Arts and Sciences and past president of the Econometric Society.

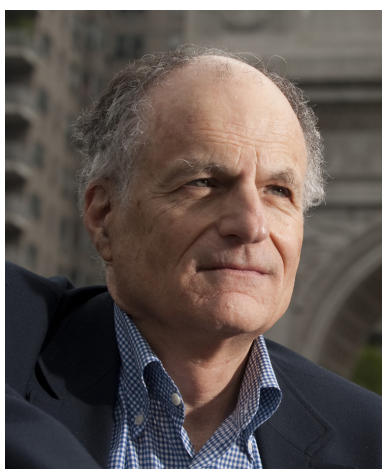

Thomas J Sargent is Professor of Economics at New York University. He was awarded the 2011 Nobel Prize in Economics, shared with Princeton University's Christopher Sims, "for their empirical research on cause and effect in the macroeconomy." He received his bachelor's degree from the University of California at Berkeley, winning the medal as the university most distinguished scholar in the Class of 1964, and obtained his $\mathrm{PhD}$ at Harvard University in 1968. He has been Professor of Economics at the University of Minnesota, the David Rockefeller Professor at the University of Chicago, and the Donald Lucas Professor of Economics at Stanford University. He has been a Senior Fellow at the Hoover Institution since 1987. He is a Fellow of the National Academy of Sciences, the American Academy of Arts and Sciences, and 
the Econometric Society. He has been President of the American Economic Association, the Econometric Society, and the Society for Economic Dynamics. In his Nobel speech in Stockholm he described himself as someone who aspires to use statistics and economic theory to understand how governments and markets can improve peoples' lives. 
This page intentionally left blank 


\section{Contents}

Foreword vii

Preface $\quad$ ix

Acknowledgements $\quad$ xiii

About the Authors $\quad$ xv

List of Figures $\quad$ xxv

List of Tables $\quad$ xxvii

1. Introduction $\quad \mathbf{1}$

1.1 Questions about Model Uncertainty .......... . 1

1.2 Ten Papers about Model Uncertainty . . . . . . . . . . 8

2. Discounted Linear Exponential Quadratic Gaussian Control 23

2.1 Cost Formulation . . . . . . . . . . . . . . . . . . . 23

2.2 Cost Recursions and Aggregator Functions . . . . . . . . 24

2.3 Infinite Horizon Costs . . . . . . . . . . . . . . . . . . . 26

2.4 Arbitrary Time-invariant Linear Control Laws . . . . . . 27

2.5 Solution to the Infinite Horizon Discounted Problem . . . . . . . . . . . . . . . . . . 29

2.6 Summary................. . . 32

3. Robust Permanent Income and Pricing 33 with Thomas D. Tallarini

3.1 Introduction . . . . . . . . . . . . . . . 33

3.2 Recursive Risk Sensitive Control . . . . . . . . . . . . 36 
3.3 Robust Permanent Income Theory _. . . . . . . . . . 42

3.4 Estimation . . . . . . . . . . . . . . . . . . 49

3.5 Asset Pricing . . . . . . . . . . . . . . 57

3.6 Quantifying Robustness from the Market Price of Risk . . . . . . . . . . . . . . . . . . 62

$3.7 \quad$ Intertemporal Mean-risk Trade-offs . . . . . . . . . . . 69

3.8 Conclusions . . . . . . . . . . . . . . . . . 75

Appendix 3.A Subgradient Inequality . . . . . . . . . . . 77

Appendix 3.B Computing Prices for State-contingent Utility . . . . . . . . . . . . . 78

Appendix 3.C Computing the Conditional Variance of the Stochastic Discount Factor . . . . . . . 79

4. A Quartet of Semigroups for Model Specification, Robustness, Prices of Risk, and Model Detection with Evan W. Anderson

4.1 Introduction . . . . . . . . . . . . . . . 83

4.2 Overview . . . . . . . . . . . . . . . . . . . 88

4.3 Mathematical Preliminaries . . . . . . . . . . . . 91

4.4 A Tour of Four Semigroups . . . . . . . . . . . . . . 97

4.5 Model Misspecification and Robust Control . . . . . . . 100

4.6 Portfolio Allocation . . . . . . . . . . . . . . . . . 107

4.7 Pricing Risky Claims . . . . . . . . . . . . . . . . . . 112

4.8 Statistical Discrimination . . . . . . . . . . . . . . . 118

4.9 Entropy and the Market Price of Uncertainty . . . . . . 129

4.10 Concluding Remarks . . . . . . . . . . . . . . . . 140

Appendix 4.A Proof of Theorem 4.5.1 . . . . . . . . . 142

5. Robust Control and Model Uncertainty 145

5.1 Introduction . . . . . . . . . . . . . . . . 145

5.2 A Benchmark Resource Allocation Problem . . . . . . . 146

5.3 Model Misspecification . . . . . . . . . . . . . 146

5.4 Two Robust Control Problems . . . . . . . . . . . . . . . 148

5.5 Recursivity of the Multiplier Formulation . . . . . . . . . 149

5.6 Two Preference Orderings . . . . . . . . . . . . 150

5.7 Recursivity of the Preference Orderings . . . . . . . . . 152

5.8 Concluding Remarks . . . . . . . . . . . . . 153 
6. Robust Control and Model Misspecification

with Gauhar A. Turmuhambetova and Noah Williams

6.1 Introduction . . . . . . . . . . . . . . . 155

6.2 Overview ........................ 159

6.3 Three Ordinary Control Problems . . . . . . . . . . 165

6.4 Fear of Model Misspecification . . . . . . . . . . . . 170

6.5 Two Robust Control Problems Defined on Sets of Probability Measures . . . . . . . . . . . . . . 171

6.6 Games on Fixed Probability Spaces . . . . . . . . . . . . 180

6.7 Sequential Timing Protocol for a Penalty Formulation . . . . . . . . . . . . . . 186

6.8 Sequential Timing Protocol for a Constraint Formulation . . . . . . . . . . . . . 190

6.9 A Recursive Multiple Priors Formulation . . . . . . . . . 197

6.10 Concluding Remarks . . . . . . . . . . . . . . . . . 201

Appendix 6.A Cast of Characters . . . . . . . . . . 203

Appendix 6.B Discounted Entropy . . . . . . . . . . . . . . 204

Appendix 6.C Absolute Continuity of Solutions . . . . . . . . 208

Appendix 6.D Three Ways to Verify Bellman-Isaacs

Condition . . . . . . . . . . . . 2 210

Appendix 6.E Recursive Stackelberg Game and Bayesian Problem . . . . . . . . . . . 213

7. Doubts or Variability?

with Francisco Barillas

7.1 Introduction . . . . . . . . . . . . . . . 217

7.2 The Equity Premium and Risk-free Rate Puzzles . . . . 219

7.3 The Choice Setting . . . . . . . . . . . . . . . . 222

7.4 A Type I Agent: Kreps-Porteus-EpsteinZin-Tallarini . . . . . . . . . . . . . . 225

7.5 A Type I Agent Economy with High Risk Aversion Attains HJ Bound . . . . . . . . . . . . . . . . 227

7.6 Reinterpretations . . . . . . . . . . . . . . . . 228

7.7 Reinterpreting Tallarini . . . . . . . . . . . . . 238

7.8 Welfare Gains from Eliminating Model Uncertainty . . . 243 
7.9 Dogmatic Bayesians and Learning . . . . . . . . . . . 251

7.10 Concluding Remarks . . . . . . . . . . . . . . 253

Appendix 7.A Formulas for Trend Stationary Model . . . . . . 254

\section{Robust Estimation and Control} without Commitment $\quad 257$

8.1 Introduction . . . . . . . . . . . . . 257

8.2 A Control Problem without Model Uncertainty . . . . . 261

8.3 Using Martingales to Represent

Model Misspecifications . . . . . . . . . . . . . . . 265

8.4 Two Pairs of Operators . . . . . . . . . . . . . 267

8.5 Control Problems with Model Uncertainty . . . . . . . . 270

8.6 The $\theta_{1}=\theta_{2}$ Case . . . . . . . . . . . . 276

8.7 Implied Worst Case Model of Signal Distortion . . . . . 284

8.8 A Recursive Multiple Priors Model . . . . . . . . . . . . 286

8.9 Risk Sensitivity and Compound Lotteries . . . . . . . . . 287

8.10 Another Example . . . . . . . . . . . . . . . 288

8.11 Concluding Remarks . . . . . . . . . . . . . . . . 290

9. Fragile Beliefs and the Price of Uncertainty 293

9.1 Introduction . . . . . . . . . . . . . . . 293

9.2 Stochastic Discounting and Risks . . . . . . . . . 296

9.3 Three Information Structures _. . . . . . . . . . . . 301

$9.4 \quad$ Risk Prices . . . . . . . . . . . . . . . . . . . . 303

9.5 A Full-Information Perspective on Agents' Learning . . . 304

9.6 Price Effects of Concerns About Robustness . . . . . . . 307

9.7 Illustrating the Mechanism . . . . . . . . . . . . . . 315

9.8 Concluding Remarks . . . . . . . . . . . . . . . . 327

Appendix 9.A Detection Error Probabilities . . . . . . . . 329

10. Beliefs, Doubts and Learning: Valuing Macroeconomic Risk

by Lars Peter Hansen

10.1 Introduction . . . . . . . . . . . . . . . . 331

10.2 Rational Expectations and Econometrics . . . . . . . 333

10.3 Statistical Precision . . . . . . . . . . . . . . . . 338

10.4 Risk Prices and Statistical Ambiguity . . . . . . . . . . . . 343

10.5 Statistical Challenges . . . . . . . . . . . . . . 347

10.6 Learning . . . . . . . . . . . . . . . . . . 352 
10.7 Beliefs and Preferences . . . . . . . . . . . . . 360

10.8 Learning and Uncertainty Premia . . . . . . . . . . 365

10.9 Extensions . . . . . . . . . . . . . . . . 375

10.10 Conclusion . . . . . . . . . . . . . . 376

11. Three Types of Ambiguity 379

11.1 Illustrative Model . . . . . . . . . . . . . . . . . . . . 385

11.2 No Concern about Robustness . . . . . . . . . . . . . 386

11.3 Representing Probability Distortions . . . . . . . . . . 392

11.4 The First Type of Ambiguity . . . . . . . . . . . . . . 394

11.5 Heterogeneous Beliefs without Robustness . . . . . . . . 401

11.6 The Second Type of Ambiguity . . . . . . . . . . . . . . . . 408

11.7 The Third Type of Ambiguity . . . . . . . . . . . . . . 409

11.8 Comparisons . . . . . . . . . . . . . . . . . . . . . 412

11.9 Numerical Example . . . . . . . . . . . . . . . . . . . . 418

11.10 Concluding Remarks . . . . . . . . . . . . . . . . 424

Appendix 11.A Some Basic Proofs . . . . . . . . . . . . 425

Appendix 11.B Example without Robustness . . . . . . . . . 426

Appendix 11.C Example with First Type of Ambiguity . . . . 428

Appendix 11.D Sensitivity to Robustness . . . . . . . . . . 430

Bibliography

431

Author Index $\quad 447$

Subject Index 
This page intentionally left blank 


\section{List of Figures}

3.1 A $(\sigma, \beta)$ locus $\ldots \ldots \ldots \ldots \ldots \ldots$

3.2 Consumption and investment . . . . . . . . . . . . 50

3.3 Two impulse response functions . . . . . . . . . . . . . . . 54

3.4 Two more impulse responses . . . . . . . . . . . . . . 55

3.5 Likelihood function . . . . . . . . . . . . . 56

3.6 Transitory and permanent endowment parts . . . . . . . . . 64

3.7 Estimated innovations . . . . . . . . . . . . . . . . 69

4.1 Dominating function . . . . . . . . . . . . . . . . . 122

4.2 An impulse response . . . . . . . . . . . . . . . . . . 133

4.3 Spectral density of consumption growth . . . . . . . . . . . 134

4.4 Drift distortion . . . . . . . . . . . . . . . . 135

4.5 Impulse response for two incomes . . . . . . . . . . . . . 137

4.6 Impulse response for persistent income . . . . . . . . . . . . 139

7.1 Hansen-Jagannathan bound . . . . . . . . . . . . . . . . 222

7.2 Detection error probabilities . . . . . . . . . . . . . 241

7.3 Risk-free rate and market price of risk . . . . . . . . . . 242

7.4 Elimination of risk and uncertainty . . . . . . . . . . . . 250

7.5 Cost of model uncertainty . . . . . . . . . . . . . . . 251

7.6 Worst-case consumption growth . . . . . . . . . . . 252

9.1 Bayesian and worst-case model probabilities . . . . . . . . . 317

9.2 Decomposition of uncertainty prices . . . . . . . . . . . . 318

9.3 Decomposition of uncertainty prices . . . . . . . . . . . . . 319

$9.4 \quad D(\iota) \Sigma(\iota) \lambda(\iota) \ldots \ldots \ldots \ldots \ldots \ldots$

9.5 Means . . . . . . . . . . . . . . . . . . 321

9.6 Means and model probabilities . . . . . . . . . . . . . . . . 323

9.7 Contributions to uncertainty prices . . . . . . . . . . . . . . 324

9.8 One-noise system . . . . . . . . . . . . . . . . . . . . . . 325 
9.9 Contributions to uncertainty prices . . . . . . . . . . 326

9.10 Unknown dynamics and unknown model . . . . . . . . . . . . 327

10.1 Mistake probabilities . . . . . . . . . . . . . . . . . . 342

10.2 Logarithm of mistake probabilities . . . . . . . . . . . . . 343

10.3 Prior and posterior probabilities . . . . . . . . . . . . . 349

10.4 Uncertainty prices . . . . . . . . . . . . . . . . 371

10.5 Time series of uncertainty prices . . . . . . . . . . 374

11.1 Four types of ambiguity . . . . . . . . . . . . . . . 380

11.2 Approximating and worst-case models . . . . . . . . . . . . 420

11.3 More approximating and worst-case models . . . . . . . . . . . 422 


\section{List of Tables}

3.1 Likelihood values . . . . . . . . . . . . . . . . . 51

3.2 Parameter estimates . . . . . . . . . . . . . . . . . 53

3.3 Median market price of risk . . . . . . . . . . . . . . 63

3.4 Worst-case shock means . . . . . . . . . . . . . . 68

3.5 Local mean-risk trade-off . . . . . . . . . . . . . . . . . . . . . . . 74

3.6 Global mean-risk trade-off . . . . . . . . . . . . . . . 74

4.1 Four semigroups . . . . . . . . . . . . . . . . 100

4.2 Uncertainty prices and detection-error probabilities . . . . . . 131

4.3 Prices of uncertainty and detection-error probabilities . . . . . 140

7.1 Sample moments . . . . . . . . . . . . . . . . . . . 221

7.2 Parameter estimates. . . . . . . . . . . . . . . . 224

7.3 Benefits of eliminating model risk and uncertainty . . . . . . 249

9.1 Risk and risk prices . . . . . . . . . . . . . . . . . . . 303

9.2 Risk and uncertainty prices . . . . . . . . . . . . . 308

10.1 Risk and uncertainty premia . . . . . . . . . . . . 369

10.2 Risk and uncertainty prices . . . . . . . . . . . 373

11.1 Drift distortions . . . . . . . . . . . . . . . . . . . . . . . . . . . . . 423

11.2 Derivatives of inflation . . . . . . . . . . . . . 423 